\title{
Pletismografía corporal: recomendaciones y procedimiento
}

\author{
Body plethysmography: Recommendations and procedure
}

\author{
Selene Guerrero-Zúñiga,* Juan Carlos Vázquez-García, ${ }^{*}$ Laura Gochicoa-Rangel,* Silvia Cid-Juárez,* \\ Rosaura Benítez-Pérez,* Rodrigo del-Río-Hidalgo,* Luis Torre-Bouscoulet*
}

*Instituto Nacional de Enfermedades Respiratorias Ismael Cosío Villegas, Ciudad de México.

\begin{abstract}
RESUMEN. La pletismografía corporal (PC) es una prueba de función respiratoria que mide la capacidad funcional residual (FRCpleth) y la resistencia específica de la vía aérea (sRaw). La PC es el estándar de oro para la medición de volúmenes pulmonares. Los resultados son útiles para confirmar la presencia de restricción pulmonar. Además, en las enfermedades obstructivas permite cuantificar la hiperinflación pulmonar y el atrapamiento aéreo. El propósito de este documento es describir el procedimiento que de forma estandarizada se sigue en el Laboratorio de Fisiología Respiratoria del Instituto Nacional de Enfermedades Respiratorias Ismael Cosío Villegas en la Ciudad de México. Este documento se apega al estándar mundial vigente; no obstante, hacemos algunas recomendaciones prácticas para la realización e interpretación de la prueba que esperamos sean de utilidad al lector.
\end{abstract}

Palabras clave: Pletismografía corporal, procedimiento, función pulmonar, volúmenes pulmonares, resistencia específica de la vía aérea.

\section{Abreviaturas:}

ATS: Asociación Americana de Tórax.

ERS: Sociedad Europea Respiratoria.

ERV: Volumen de reserva espiratoria.

FRC: Capacidad funcional residual.

Gaw: Conductancia de la vía aérea.

IRV: Volumen de reserva inspiratoria.

IC: Capacidad inspiratoria.

LIN: Límite inferior de la normalidad.

LSN: Límite superior de la normalidad.

Raw: Resistencia de la vía aérea.

RV: Volumen residual.

sRawtot: Resistencia específica total.

sRaweff: Resistencia específica efectiva.

\section{Correspondencia:}

\section{Dr. Luis Torre-Bouscoulet}

Instituto Nacional de Enfermedades Respiratorias Ismael Cosío Villegas.

Calzada de Tlalpan Núm. 4502,

Colonia Sección XVI, 14080, Ciudad de México.

Teléfono: +52 -55 -54871700, ext. 5160

Correo electrónico: luistorreb@gmail.com

Trabajo recibido: 13-IX-2016; aceptado: 20-IX-2016.
ABSTRACT. Body plethysmography (BP) is a pulmonary function test that measures functional residual capacity (FRCpleth) and specific airway resistance (sRaw). BP currently constitutes the gold standard for measuring pulmonary volumes. Results of this test are effective in confirming the presence of pulmonary restriction, while in cases of obstructive disease they permit quantification of pulmonary hyperinflation and air trapping. The objective of this manuscript is to describe the standardized procedure for BP testing followed at the Respiratory Physiology Laboratory at Mexico City's National Institute of Respiratory Diseases (Instituto Nacional de Enfermedades Respiratorias Ismael Cosío Villegas). While respecting current international standards, the article includes some practical recommendations for performing and interpreting this test that we hope readers will find useful.

Keywords: Body plethysmography, procedure, lung function, lung volume, specific airway resistance.

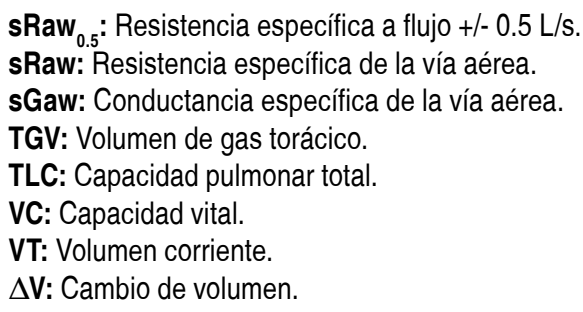

\section{INTRODUCCIÓN}

La pletismografía corporal (PC) es una prueba de función respiratoria que mide el volumen de gas intratorácico (TCV) y la resistencia específica de la vía aérea (sRaw). ${ }^{1,2}$ Las mediciones obtenidas en la PC dependen de la distensibilidad y elasticidad de la caja torácica y del parénquima pulmonar, así como de la integridad de la musculatura respiratoria. Aunque existen varias técnicas de medición, la PC es el estándar de oro para la medición de volúmenes pulmonares. El propósito de este manuscrito es revisar el estándar vigente para la realización de la prueba de PC, así como emitir recomendaciones prácticas para su interpretación. Esperamos que este documento contribuya a mejorar la 
calidad y estandarización de la PC en México y otros países de Latinoamérica.

\section{DEFINICIÓN}

La PC es una prueba de mecánica de la respiración cuya principal medición directa es la capacidad funcional residual (FRCpleth) ${ }^{1,2}$ que corresponde al volumen de gas intratorácico (ITGV o TGV) medido al final de la espiración a volumen corriente estable. Se realiza adicionalmente una medición de capacidad vital lenta (VC), volumen corriente (VT), capacidad inspiratoria (IC) y volumen de reserva espiratorio (ERV). Esas mediciones permiten calcular la capacidad pulmonar total (TLC) y el volumen residual (RV). ${ }^{1,2}$

El método se desarrolló con base en los conceptos descritos por Bert, Gad y Pflüger en el siglo XIX y fue realizada por primera vez por DuBois en la década de $1950 .{ }^{2,3} \mathrm{La}$ medición está basada en la Ley de Boyle-Mariotte que señala que el volumen de un gas es inversamente proporcional a la presión a la que está sujeto en condiciones isotérmicas; por lo tanto, durante la compresión de un gas en estas condiciones, el producto de la presión por el volumen permanece constante. ${ }^{1,3}$

Los parámetros funcionales que nos brinda la PC son: ${ }^{4,5}$

a) Volumen corriente (VT): es el volumen de aire que es inspirado y espirado con cada ciclo durante una respiración normal. b) Volumen residual (RV): es el volumen de aire remanente en los pulmones después de una exhalación máxima.

c) Volumen de reserva espiratorio (ERV): es el máximo volumen de aire que puede ser exhalado desde el nivel previo de FRC.

d) Volumen de reserva inspiratorio (IRV): es el mayor volumen de aire que se puede inhalar desde un nivel previo al final de la inspiración de un ciclo respiratorio normal.

e) Capacidad inspiratoria (IC): es el mayor volumen de aire que se puede inspirar desde un nivel previo de FRC, corresponde a la suma del VT y el IVR.

f) Capacidad residual funcional (FRCpleth o ITGV): Es el volumen de aire dentro de los pulmones al final de la espiración de un ciclo respiratorio normal, corresponde a la suma del ERV y RV. Está determinada por el balance entre la retracción elástica del pulmón y el retroceso elástico de la pared del tórax mientras los músculos respiratorios se encuentran en reposo.

g) Capacidad vital (VC): es el volumen máximo de aire que puede exhalarse desde capacidad pulmonar total hasta llegar a volumen residual (capacidad vital exhalatoria); o bien, inhalarse desde volumen residual hasta capacidad pulmonar total (capacidad vital inhalatoria). Corresponde a la suma de los volúmenes desplazables VT, IRV y ERV.

h) Capacidad pulmonar total (TLC): es el mayor volumen de aire contenido en los pulmones después de una inspiración máxima siendo la suma de los cuatro volúmenes pulmonares (Figura 1).

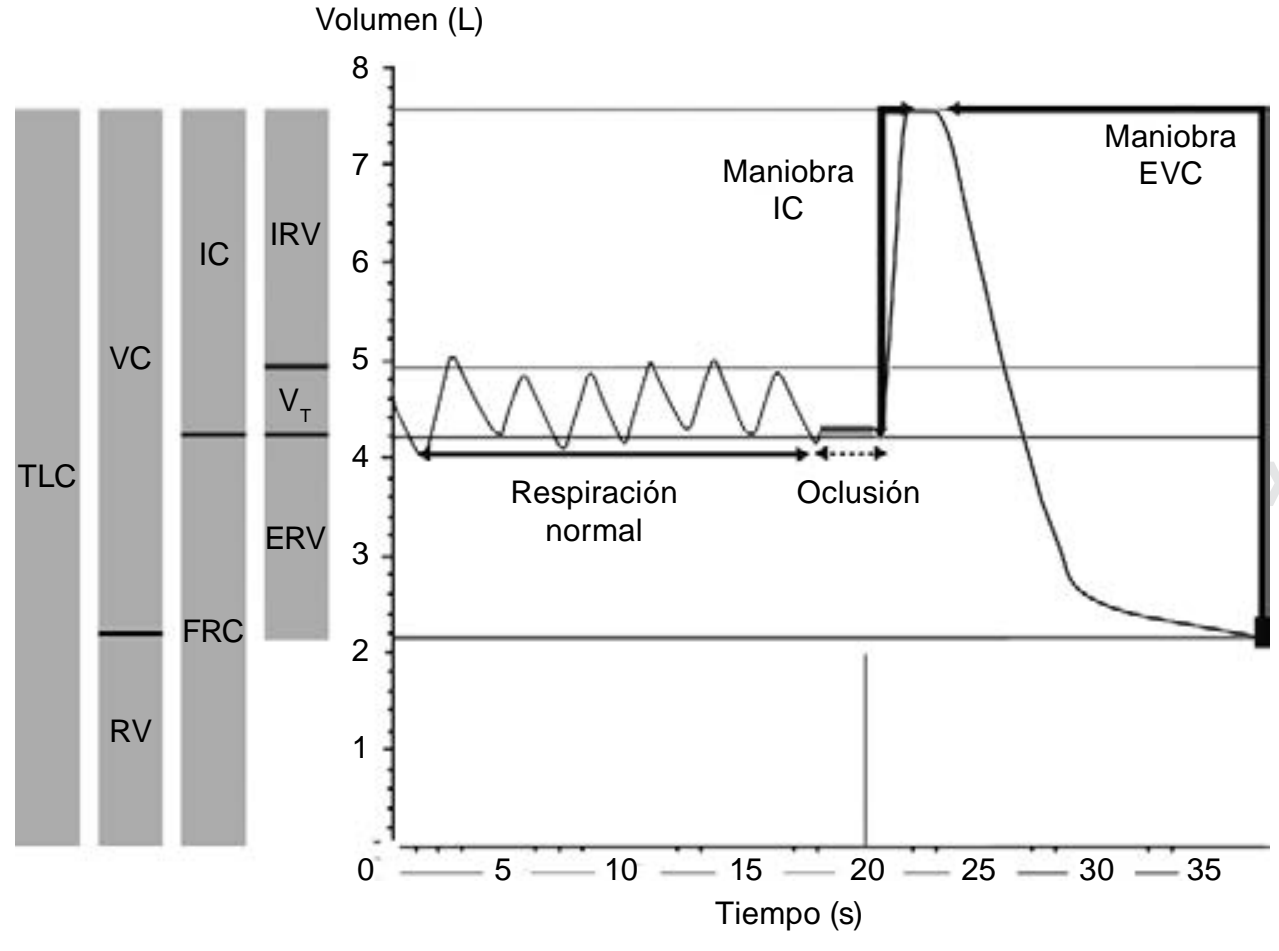

Figura 1:

Maniobra de pletismografía para volúmenes pulmonares. Gráfico volumen-tiempo con desglose de volúmenes y capacidades, inicia con respiración normal para medición de volumen corriente $\left(\mathrm{V}_{\mathrm{T}}\right)$ hasta mantener nivel de capacidad funcional residual estable (FRC) seguida de oclusión para medición de FRCpleth la cual debe ocurrir a menos de $200 \mathrm{~mL}$ del nivel de FRC estabilizado (área sombreada), posteriormente inspiración máxima, para medición de capacidad inspiratoria (IC), finaliza con maniobra de capacidad vital espiratoria relajada (EVC) que alcance meseta último segundo con cambio de volumen $<25 \mathrm{~mL}$. En esta secuencia el equipo calcula la capacidad pulmonar total $(T L C=$ FRCpleth + IC) y el volumen residual $(\mathrm{RV}=\mathrm{TLC}-\mathrm{EVC})$. 
i) Resistencia especifica en las vías respiratorias (sRaw): es la relación entre el flujo medido en la boca y el cambio de volumen medido en la cabina durante respiración a VT. ${ }^{1}$

j) Resistencia en vías respiratorias (Raw): indica la presión alveolar necesaria para generar un flujo de $1 \mathrm{~L} / \mathrm{s}$, se calcula ajustando la sRAW a la FRCpleth y al VT como sigue Raw $=$ sRaw $/($ FRCpleth $+\mathrm{VT} / 2)$; se mide en $\mathrm{CmH}_{2} \mathrm{O} / \mathrm{L} /$ seg. ${ }^{2}$

k) Conductancia de las vías respiratorias (Gaw): es el recíproco de la sRAW (1/sRAW) medida en $\left(\mathrm{L} / \mathrm{seg} / \mathrm{cmH}_{2} \mathrm{O}\right)$; corresponde a la conductancia. Este valor mantiene una relación lineal con el volumen. ${ }^{1}$

\section{Indicaciones}

a) Confirmación y cuantificación de restricción pulmonar.

b) Confirmación y cuantificación de hiperinflación pulmonar y atrapamiento aéreo especialmente en pacientes con disnea desproporcionada al grado de obstrucción por $\mathrm{FEV}_{1}$; o bien, durante la evaluación preoperatoria de cirugía de reducción de volumen. ${ }^{6}$

c) Monitorización y vigilancia de enfermedad con fines clínicos o de investigación. ${ }^{2,5,7}$

\section{Contraindicaciones (aplican para la mayoría de las pruebas de función respiratoria)}

Las contraindicaciones son relativas e incluyen:

a) Infarto reciente ( $<4$ semanas).

b) Insuficiencia cardíaca.

c) Inestabilidad cardiovascular.

d) Taquicardia reposo (FC > $130 \mathrm{lpm}$ ).

e) Cirugía de tórax, abdomen $<4$ semanas o cirugía de ojos u oído en $<8$ semanas.

f) Tuberculosis pulmonar activa.

g) Influenza.

h) Hemoptisis.

i) Aneurismas (grandes arterias, cerebrales).

j) Embarazo avanzado o complicado.

k) Salud precaria.

l) Traqueostomía

m) Sonda pleural.

n) Requerimiento continuo de oxígeno suplementario que no se pueda suspender durante la prueba.

o) Condiciones del paciente que no permitan introducirlo a la cabina, como claustrofobia, parálisis corporal, soluciones parenterales o aditamentos médicos que no puedan introducirse a la cabina del pletismógrafo. ${ }^{8}$

\section{Requerimiento del pletismógrafo}

El pletismógrafo es una cámara hermética de tamaño suficiente para que se pueda introducir una persona. Su capacidad es en general, entre 600 y 1,000 litros. El equipo y software deben cumplir con las especificaciones técnicas emitidas por la ATS/ERS 2005 con los siguientes requerimientos: ${ }^{4}$

I. Transductores de presión capaces de medir más de 50 $\mathrm{cmH}_{2} \mathrm{O}$ y con frecuencia de respuesta de al menos 8 $\mathrm{Hz}$. La frecuencia de respuesta debe ser mínimo cinco veces superior a la frecuencia respiratoria durante la maniobra de ITGV que no debe rebasar $1.5 \mathrm{~Hz}$ (90 respiraciones por minuto). ${ }^{4}$

II. Los sensores para medir flujo o volumen (neumotacógrafo) deben cumplir con todos los estándares para espirómetros (ATS/ERS 2005): capacidad mínima de medición de volumen de 0.5 a $8.00 \mathrm{~L}$ (con exactitud de $\pm 3 \%$, medido con jeringa de $3.00 \mathrm{~L}$ ), flujos de $0 \mathrm{a}$ $14 \mathrm{~L} / \mathrm{s}$ y tiempo de registro de al menos 30 segundos. ${ }^{4}$

III. Los transductores de presión de la cabina del pletismógrafo deben tener una exactitud de $\pm 0.2 \mathrm{CmH}_{2} \mathrm{O} .{ }^{4}$

IV. Pantalla para presentar la gráfica de volumen-tiempo, presión-cambio de volumen, flujo-cambio de volumen. ${ }^{4}$

V. Impresión del informe el cual deberá incluir datos del paciente, valores de referencia y las principales mediciones en pletismografía: FRCpleth, RV, VC, IC, ERV, TLC, RV/ TLC, Rtot, FR durante medición de FRCpleth, cambio de volumen $(\Delta \mathrm{V})$, así como la gráfica volumen tiempo, presión-cambio de volumen y flujo-cambio de volumen. ${ }^{4}$

\section{Equipos y consumibles: ${ }^{8}$}

a) Computadora e impresora, de acuerdo con los requerimientos del equipo de pletismografía.

b) Báscula, estadímetro y cinta métrica (para medición de extensión de brazos, cuando se requiera).

c) Termómetro ambiental con una precisión de $1^{\circ} \mathrm{C}$.

d) Boquillas con filtros en línea desechables con eficiencia de $>99 \%$ para filtración de virus, bacterias y micobacterias; espacio muerto $<100 \mathrm{~mL}$ y resistencia menor $1.5 \mathrm{cmH}_{2} \mathrm{O}$ a un flujo de $6 \mathrm{~L} / \mathrm{s}$.

e) Pinza nasal.

f) Aditamentos para control de infecciones cruzadas.

I. Acceso a lavado de manos y gel-alcohol.

II. Guantes desechables.

III. Cubre-boca quirúrgico para protección general y cuando se requiera cubre-boca N95 con fuga menor al $10 \%$ y una eficiencia de filtrado de $>95 \%$ a un flujo de $50 \mathrm{~L} / \mathrm{min}$.

IV. Anteojos de protección.

\section{Indicaciones al paciente antes de la prueba ${ }^{8}$}

a) Evitar tabaquismo 2 horas antes.

b) Evitar el uso de prendas restrictivas de tórax, como chalecos, corsés o ropa muy apretada. 
c) Mantener su medicación de base.

d) No se requiere de ayuno para la prueba, pero se recomienda alimentación ligera cuatro horas antes.

e) Se debe evitar ejercicio intenso antes de la prueba.

f) Es recomendable aplicar un cuestionario breve con antecedente de tabaquismo, exposición a humos o polvos, historia de enfermedades o trauma torácicopulmonar.

\section{Preparación del equipo antes de la prueba}

a) Calibración: procedimiento por el cual se establece una relación entre el volumen o flujo medido por el sensor y el flujo o volumen real del calibrador (jeringa). Esta maniobra se refiere a un ajuste de ganancia eléctrica del dispositivo. ${ }^{9}$

b) Verificación de calibración: concepto diferente al de calibración. Este procedimiento se usa para validar que el sensor de flujo se encuentra dentro de los límites de calibración (exactitud), $\pm 0.3 \%$. Si el dispositivo falla, la verificación debe repetirse. En caso de fracaso repetido, el equipo debe enviarse a mantenimiento o revisión. ${ }^{9}$

c) Procedimiento:

I. Encendido y calentamiento del equipo por 30 minutos. ${ }^{4}$

II. El sensor de flujo del pletismógrafo deberá ser calibrado para volumen en forma diaria y realizar revisión semanal de linealidad utilizando una jeringa de $3.00 \mathrm{~L}$ la cual deberá contar con una exactitud de $\pm 0.5 \%$ de volumen absoluto $(15 \mathrm{~mL})$ sin daño o golpes, asegurando una conexión firme y hermética con el sensor de flujo. Almacene los datos de la calibración. Las mediciones deben fluctuar entre 2.91 y $3.09 \mathrm{~L}( \pm 3 \%) .{ }^{9}$

III. La rutina de calibración debe seguir las recomendaciones del fabricante. ${ }^{4}$

IV. Revisión de resistencia del obturador la cual debe ser mínima.

V. Revisión diaria de fugas del sistema y cierre hermético de la puerta.

VI. Alineación de los transductores. En general el transductor de presión de la cabina se calibra en términos de TGV al introducir y retirar $50 \mathrm{~mL}$ de aire con una jeringa automatizada. ${ }^{1}$

VII. De forma semanal o máximo mensual, realizar control biológico. Las variaciones $>10 \%$ en FRC y TLC o $>20 \%$ para RV sugieren errores de medición. ${ }^{4}$

\section{Preparación del paciente antes de la prueba}

a. El técnico que realiza la prueba deberá recibir y presentarse con el paciente. b. Verificar la solicitud del paciente, con número de registro y fecha del estudio.

c. Confirmar con el paciente o familiar del paciente que sus datos sean correctos (nombre y fecha de nacimiento).

d. En caso de que el paciente no hable español, deberá acompañarlo un intérprete para explicarle el procedimiento.

e. Explicar al paciente el objetivo de la prueba. La frase recomendada es la siguiente:

«La pletismografía es una prueba que sirve para medir el tamaño de sus pulmones, se parece mucho a la espirometría, pero para realizarla tiene que estar dentro de esta cabina cerrada durante aproximadamente 15 minutos. No se preocupe por estar dentro de la cabina, usted no sentirá malestar y yo lo estaré vigilando e instruyendo en todo momento.»

f. Registrar el consumo de tabaco, el ejercicio físico intenso antes de la prueba y el uso de broncodilatadores.

g. El técnico deberá reportar a su supervisor si detecta contraindicaciones de la prueba, quien deberá evaluar la pertinencia del estudio y supervisar la ejecución de la misma.

h. La estatura se mide en centímetros $(\mathrm{cm})$ y con el individuo sin zapatos, en posición completamente erguida, talones juntos y mirando al frente. Es conveniente utilizar un estadímetro de pared. En los pacientes que no puedan mantenerse de pie o sufran de deformidad de caja torácica, se puede usar la extensión de los brazos como una estimación de la estatura. Se mide la extensión entre el extremo de los dedos medios de cada mano. La estatura se estima para hombres como extensión de brazos en centímetros dividido entre 1.033 y para mujeres dividida entre 1.011, estos valores ajustan bien para estatura entre 150 y $190 \mathrm{~cm}$, de los 10 a los 60 años de edad; otras ecuaciones han sido propuestas para agregar el efecto de la edad y grupo étnico, o bien existen fórmulas basadas en la altura del piso a la rodilla. ${ }^{8,10-12}$

i. El peso se mide en una báscula calibrada y se registra en kilogramos ( $\mathrm{kg}$ ) en unidades cerradas al 0.5 kg más cercano. El peso no es utilizado en la mayoría de las ecuaciones de referencia para calcular valores normales, pero es útil para fines de interpretación y permite al equipo calcular el volumen del paciente.

j. Iniciar el software programa de datos del paciente en el pletismógrafo y registrar los datos, nombre completo, etnicidad, fecha de nacimiento, género, parámetros antropométricos (peso y estatura).

k. Ajustar la silla que se encuentra dentro de la cabina, permitiendo que el paciente permanezca sentado con 
el tórax y cuello en posición recta y con ambos pies apoyados sobre el piso. Adecuar la altura del sensor de flujo para que el paciente alcance la boquilla sin extender o flexionar el cuello.

I. Explicar y demostrar la maniobra de pletismografía. El técnico demuestra cómo colocar la boquilla; sujetar con los dientes (sin morder), sellar con los labios, sostener las mejillas con ambas manos y colocación de la pinza nasal. Verificar que la expansión del tórax no se vea limitada por el uso de fajas, corsé, etc. y explicar que la puerta se mantendrá cerrada en todo momento durante la medición. Antes de iniciar una prueba, asegurar que el paciente se encuentra cómodo y entiende completamente las indicaciones para realizar la prueba.

\section{Maniobra de pletismografía}

a. Explicar el procedimiento que se va a realizar, enfatizando las siguientes indicaciones al paciente:

I. Deberá mantenerse sentado dentro de la cabina, con el tórax y cuello en posición recta y con ambos pies apoyados sobre el piso.

II. Puede mover el sensor hacia adelante o atrás para tomar la boquilla sin flexionar o extender el cuello.

III. Antes de iniciar la medición coloque la pinza en su nariz.

IV. Deberá sostener sus mejillas con ambas manos (demostrar).

V. Recuerde que la boquilla deberá sostenerse con los dientes sin morderla o meter la lengua y deberá sellar los labios alrededor de la misma.

VI. Deberá respirar normalmente a través de la boquilla de 3 a 10 respiraciones, posteriormente la boquilla será obturada. No se asuste, sólo dura algunos segundos durante los cuales debe respirar rápido y superficial, aproximadamente una respiración por segundo. Al abrirse la válvula inspire profundamente hasta llenar sus pulmones y posteriormente espire en forma relajada hasta que le indique que la medición ha terminado.

b. Una vez explicado el procedimiento, cierre la puerta de la cabina y espere un minuto a que se estabilice la presión y la temperatura dentro de la cabina.

c. Supervisar la adecuada colocación de la boquilla y pinza nasal, así como el adecuado soporte de mejillas con las manos.

d. Solicitar que respire tranquilo, obteniendo de 3 a 10 respiraciones en volumen corriente, esperando se estabilice el nivel del volumen al final de cada espiración (FRC estable).

e. Ocluir la válvula al final de una espiración cuando el paciente se encuentre en el nivel de FRC, indique al paciente respirar con frecuencia de $.5 \mathrm{a} 1.5 \mathrm{~Hz}$ o de una respiración por segundo (30 a 90 respiraciones por minuto) en forma suave.

f. Al abrirse la válvula de obturación solicite al paciente una inspiración máxima y posteriormente espiración relajada hasta lograr meseta por lo menos de un segundo con cambio de volumen $<25 \mathrm{~mL}$.

g. En pacientes con disnea grave que no pueden completar la maniobra de VC inmediatamente después de las maniobras de ITGV, pueden realizar algunas respiraciones en volumen corriente y después intentar la maniobra de VC.

h. Verifique que la medición de FRCpleth (ITGV) genere curvas de ITGV casi rectas y superpuestas entre ellas, estando dentro de los rangos de calibración de presión de los transductores $\left( \pm 10 \mathrm{cmH}_{2} \mathrm{O}\right.$ o $1.3 \mathrm{kPa}$ ) y corregir en caso necesario (Figura 2).

i. Obtener una serie de 3 a 5 maniobras de ITGV-VC que deben cumplir los siguientes criterios de aceptabilidad (Figuras 1 y 2):

I. El nivel de FRC se mantiene estable durante las respiraciones a volumen corriente. ${ }^{4}$

II. La diferencia de volumen (delta de volumen $\Delta \mathrm{V}$ ) entre el nivel de FRC y el nivel de oclusión es menor de $200 \mathrm{~mL} .{ }^{4}$

III. La frecuencia respiratoria durante la obturación de la válvula es entre 0.5 a $1.5 \mathrm{~Hz}$ (30 y 90 respiraciones por minuto). ${ }^{4}$

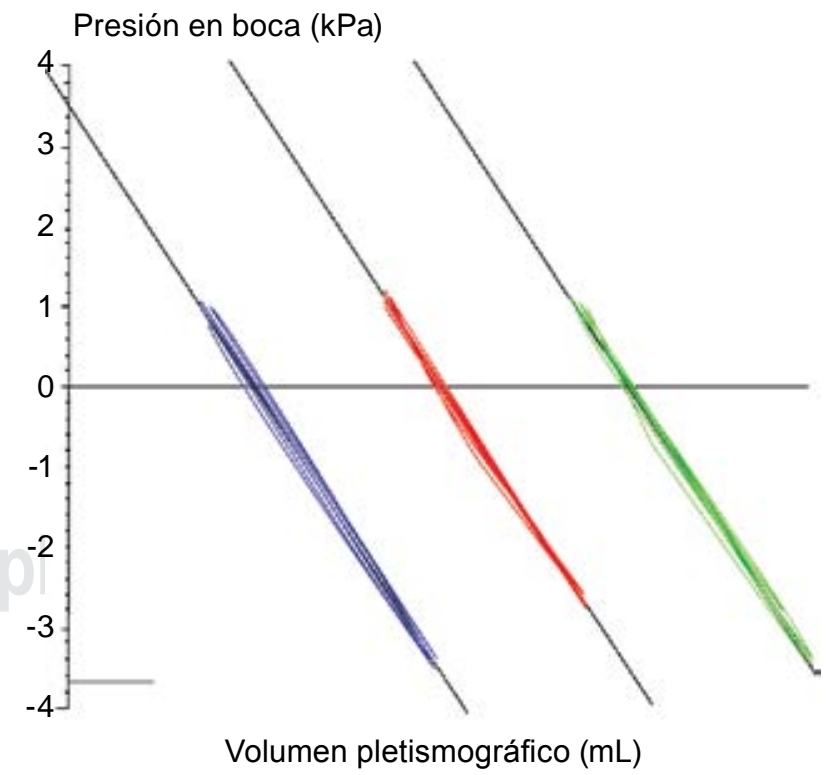

Figura 2: Gráfica presión en boca-cambio de volumen pletismográfico durante la medición de FRCpleth. Se observan de 3 a 5 líneas (FR durante la medición 0.5-1.5 Hz), las curvas son regulares, sin artefactos y con histéresis mínima entre la inspiración (valores negativos Pboca) y espiración (valores positivos Pboca), visibles ambos extremos con valores $<1.3 \mathrm{KPa}\left(<10 \mathrm{cmH}_{2} \mathrm{O}\right)$ y se mantienen paralelos a la línea de medición. 
IV. En la gráfica de ITGV (presión-volumen) se observan 3 a 5 respiraciones en cada maniobra. ${ }^{4}$

V. Las curvas de ITGV son regulares y sin artefactos, con histéresis mínima entre la inspiración y la espiración. ${ }^{4}$

VI. Se deben observar los dos extremos de la curva y con esfuerzos menores a $10 \mathrm{cmH}_{2} \mathrm{O}$ o $1.3 \mathrm{kPa}^{4}{ }^{4}$

VII. La pendiente de las curvas registradas es paralela a la línea de medición. ${ }^{4}$
VIII. La medición de VC es aceptable en espiración que alcance meseta de al menos un segundo con un cambio de volumen $<25 \mathrm{~mL}$. ${ }^{4}$

j. Las mediciones deben contar con los siguientes criterios de repetibilidad:

1. Varianza menor al $5 \%$ en las maniobras de ITGV aceptables. El cálculo de la varianza se obtiene: [(FRCpleth mayor-FRCpleth menor)/FRCpleth promedio] $* 100 .{ }^{4}$

\begin{tabular}{|c|c|c|}
\hline \multicolumn{3}{|c|}{$\begin{array}{l}\text { Nombre de la institución } \\
\text { Informe de pletismografía corporal }\end{array}$} \\
\hline Nombre: & Edad: & Fecha: \\
\hline Género: & Peso: & Referencia: \\
\hline
\end{tabular}

\begin{tabular}{|c|c|c|c|c|c|c|c|}
\hline & & Pred & Mejor & Act1 & Act2 & Act3 & $\%$ Pred \\
\hline ITGV & (L) & & & & & & \\
\hline RV & $(\mathrm{L})$ & & & & & & \\
\hline VC & (L) & & & & & & \\
\hline IC & (L) & & & & & & \\
\hline ERV & (L) & & & & & & \\
\hline TLC & $(\mathrm{L})$ & & & & & & \\
\hline RV/TLC & $(\%)$ & & & & & & \\
\hline $\mathrm{sR}_{\mathrm{tot}}$ & $(\mathrm{kPa} / \mathrm{s})$ & & & & & & \\
\hline$s R_{\text {eff }}$ & $(\mathrm{kPa} / \mathrm{s})$ & & & & & & \\
\hline $\mathrm{sRaw}_{0.5}$ & $(\mathrm{kPa} / \mathrm{s})$ & & & & & & \\
\hline FRITGV & $(1 / \mathrm{min})$ & & & & & & \\
\hline Delta volumen & $(\mathrm{L})$ & & & & & & \\
\hline
\end{tabular}
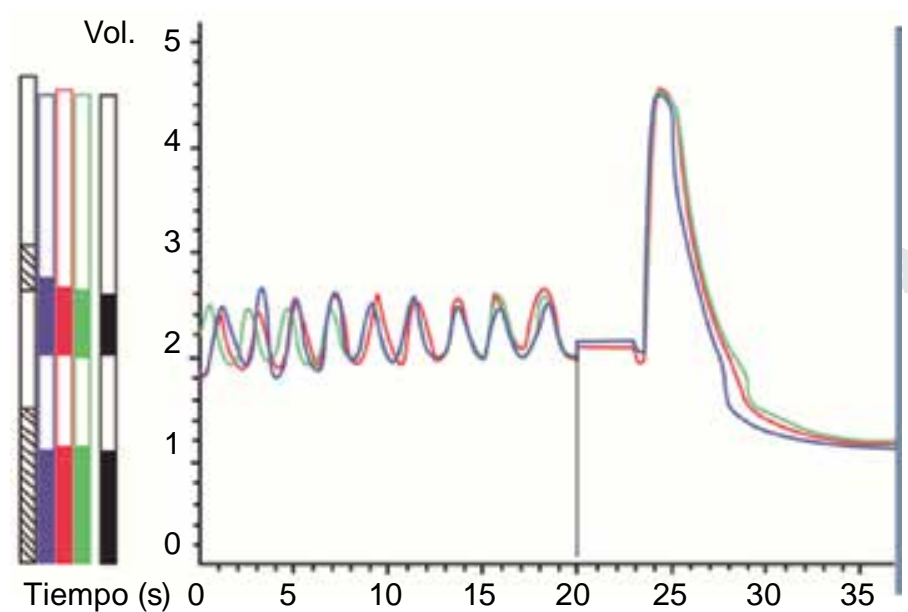

Presión en boca $(\mathrm{kPa})$

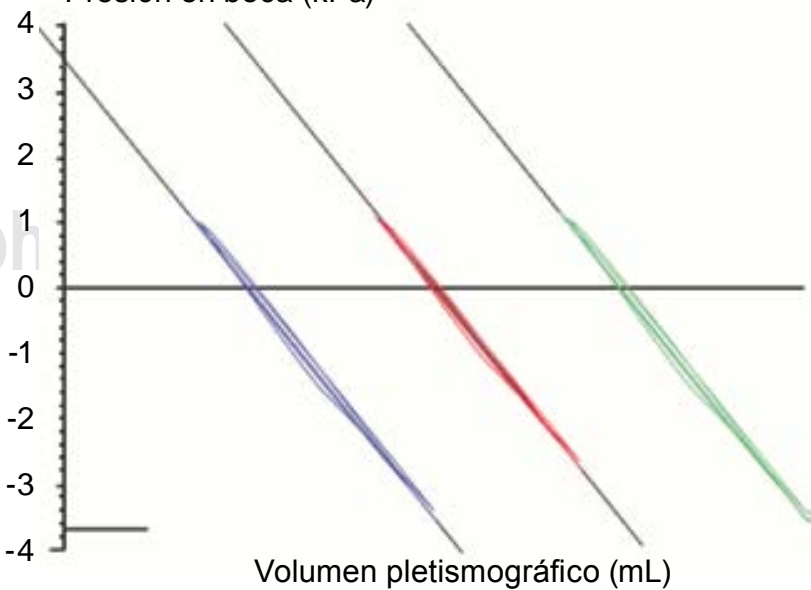

Figura 3: Informe propuesto de pletismografía corporal. Incluye gráfico volumen-tiempo y presión-volumen pletismográfico, así como valores numéricos para las principales mediciones. 
Tabla 1: Ecuaciones de referencia para volúmenes pulmonares.

\begin{tabular}{|c|c|c|c|c|c|c|}
\hline Autor & $\begin{array}{c}\text { Sujetos } \\
\text { incluidos }\end{array}$ & Edad & Ecuación & & IC $90 \%$ & RSD \\
\hline \multirow[t]{3}{*}{ Zapletal'13 } & \multirow[t]{3}{*}{174} & & Niños & $\begin{array}{l}\text { TLC } m L 9.96^{*} 10^{-3}{ }^{*} \mathrm{E}^{2.5698} \\
\text { FRC } \mathrm{mL} 3.22^{*} 10^{-3}{ }^{*} \mathrm{E}^{2.6523} \\
\text { RV } \mathrm{mL} 21.06^{*} 10^{-3} \mathrm{E}^{2} \mathrm{E}^{2.1114}\end{array}$ & $\begin{array}{l}90-111 \% p \\
87-116 \% p \\
73-137 \% p\end{array}$ & $\begin{array}{l}0.064 \\
0.088 \\
0.191\end{array}$ \\
\hline & & & Niñas & $\begin{array}{l}\text { TLC } m L 9.17^{*} 10^{-3}{ }^{*} \mathrm{E}^{2.5755} \\
\text { FRC } \mathrm{mL} 3.70^{*} 10^{-3}{ }^{*} \mathrm{E}^{2.6149} \\
\text { RV } \mathrm{mL} 21.06^{*} 10^{-3}{ }^{*} \mathrm{E}^{2.1114}\end{array}$ & $\begin{array}{l}87-115 \% p \\
82-123 \% p \\
73-137 \% p\end{array}$ & $\begin{array}{l}0.083 \\
0.124 \\
0.191\end{array}$ \\
\hline & & & Niños y niñas & RV\%TLC 21.7 & $12-31 \%$ & 5.70 \\
\hline \multirow[t]{2}{*}{ Quanjer ${ }^{13}$} & & & Hombres & $\begin{array}{l}\text { TLC (L) } 7.99^{\star} E-7.08 \\
\text { RV (L) } 1.31^{*} E+0.022^{\star} A-1.23 \\
\text { FRC (L) } 2.34^{\star} E+0.01^{\star} A-1.09 \\
\text { RV/TLC (\%) } 14.0+0.39^{\star} A\end{array}$ & $\begin{array}{l} \pm 1.15(\mathrm{~L}) \\
\pm 0.80(\mathrm{~L}) \\
\pm 1.18(\mathrm{~L}) \\
\pm 9.0(\%)\end{array}$ & $\begin{array}{l}0.70 \\
0.41 \\
0.60 \\
5.46\end{array}$ \\
\hline & & & Mujeres & $\begin{array}{l}\text { TLC (L) } 6.60^{\star} E-5.79 \\
\text { RV (L) } 1.81^{\star} E+0.016^{\star} A-2.00 \\
\text { FRC (L) } 2.24^{\star} E+0.001^{\star} A-1.00 \\
\text { RV/TLC (\%) } 19.0+0.34^{\star} A\end{array}$ & $\begin{array}{l} \pm 0.99(\mathrm{~L}) \\
\pm 0.69(\mathrm{~L}) \\
\pm 0.98(\mathrm{~L}) \\
\pm 9.6(\%)\end{array}$ & $\begin{array}{l}0.60 \\
0.35 \\
0.50 \\
5.83\end{array}$ \\
\hline \multirow[t]{2}{*}{ Roca et al. ${ }^{14}$} & \multirow[t]{2}{*}{482} & \multirow[t]{2}{*}{$20-70$} & Hombres & $\begin{array}{l}\text { TLC (L) 92.687E+8.301A -9129 } \\
\text { RV (L) 22.618E + 20.664A -2688 } \\
\text { FRC (L) } 57.878 E+10.148 \mathrm{~A}-6766 \\
\text { RV/TLC (\%) } 0.277 \mathrm{~A}+17.35\end{array}$ & & $\begin{array}{l}808 \\
497 \\
675 \\
5.44\end{array}$ \\
\hline & & & Mujeres & $\begin{array}{l}\text { TLC (L) 63.661E }-4775 \\
\text { RV (L) } 11.331 E+11.651 A-562 \\
\text { FRC (L) } 36.024 E-2847 \\
\text { RV/TLC - } 0.157 E+0.257 A+47.6\end{array}$ & & $\begin{array}{l}584 \\
412 \\
504 \\
6.35\end{array}$ \\
\hline \multirow[t]{2}{*}{ Manzke et al..$^{15}$} & \multirow[t]{2}{*}{$\begin{array}{c}400 \\
(187 F)\end{array}$} & \multirow[t]{2}{*}{$6-16$} & Niños & $\begin{array}{l}\mathrm{nlog}(\mathrm{TLC})=-1.3191+1.7383 \mathrm{E} \\
\mathrm{RV}=0.0046+0.1473 \mathrm{E}^{3}+2.8681 / \mathrm{A} \\
\mathrm{nlog}(\mathrm{FRC})=-1.8195+1.6779 \mathrm{E} \\
\mathrm{nl} \log (\mathrm{RV} / \mathrm{TLC})=2.4628+6.5376 / \mathrm{A}\end{array}$ & & $\begin{array}{l}0.10 \\
0.28 \\
0.12 \\
0.31\end{array}$ \\
\hline & & & Niñas & $\begin{array}{l}\text { nlog(TLC) }=-1.2940+1.7021 E \\
R V=-0.2189+0.2042 E^{3}+3.6015 / A \\
n \log (F R C)=-2.0159+1.7942 E \\
n \log (R V / T L C)=2.6911+4.7997 / A\end{array}$ & & $\begin{array}{l}0.11 \\
0.25 \\
0.12 \\
0.31\end{array}$ \\
\hline \multirow[t]{2}{*}{ García-Rio et al. ${ }^{16}$} & \multirow[t]{2}{*}{$\begin{array}{c}321 \\
\text { (189F) }\end{array}$} & \multirow[t]{2}{*}{$65-85$} & Hombres & $\begin{array}{l}\text { TLC (L) } 2.321-0.00000309 \mathrm{~A}^{3}+ \\
\quad 0.0000009765 \mathrm{E}^{3} \\
\text { RV (L) } 0.315+0.0000003551 \mathrm{E}^{3} \\
\text { FRC (L) }-1.215+0.02434 \mathrm{~A}+0.0000005659 \mathrm{E}^{3} \\
\text { RV/TLC (\%) } 11.59+0.314 \mathrm{~A} \\
\text { sRaw, } \mathrm{kPa} / \mathrm{s}-0.204+0.01949 \mathrm{~A}\end{array}$ & & $\begin{array}{l}0.8001 \\
0.5369 \\
0.4865 \\
6.8354 \\
0.5008\end{array}$ \\
\hline & & & Mujeres & $\begin{array}{l}\text { TLC (L) } 12.383-0.243 \mathrm{~A}+0.00001271 \mathrm{~A}^{3}+ \\
\quad 0.0001824 \mathrm{E}^{2} \\
\text { RV (L) }-1.039+0.01678 \mathrm{E} \\
\text { FRC (L) } 0.563+0.0000004998 \mathrm{E}^{3} \\
\text { RV/TLC (\%) } 29.404+0.00002356 \mathrm{~A}^{3} \\
\text { sRaw KPa/s } 0.542+0.02527 \mathrm{~A}\end{array}$ & & $\begin{array}{l}0.5223 \\
0.4168 \\
0.4083 \\
7.3748 \\
0.6996\end{array}$ \\
\hline
\end{tabular}


Tabla 1 (continuación): Ecuaciones de referencia para volúmenes pulmonares.

\begin{tabular}{|c|c|c|c|c|c|c|}
\hline Autor & $\begin{array}{l}\text { Sujetos } \\
\text { incluidos }\end{array}$ & Edad & Ecuación & & IC 90\% & RSD \\
\hline \multirow[t]{2}{*}{ Koch et al. ${ }^{17}$} & \multirow[t]{2}{*}{$\begin{array}{c}686 \\
(411 F)\end{array}$} & \multirow[t]{2}{*}{$25-85$} & Hombres & $\begin{array}{l}\text { TLC (L) }-11.9103+0.0038 A+0.1073 E \\
\text { RV (L) -3.6871+0.0264A+0.0268E } \\
\text { FRC (L) }-10.419+0.0172 A+0.092 E-0.0329 P \\
\text { RV/TLC 38.788 }-0.2115 A+0.0055 A 2-0.0588 E\end{array}$ & & \\
\hline & & & Mujeres & $\begin{array}{l}\text { TLC (L) }-5.8147+0.0528 \mathrm{~A}-0.0005 \mathrm{~A}^{2}+0.0611 \mathrm{E} \\
\text { RV (L) }-2.8192+0.0253^{\mathrm{a}}+0.0249 \mathrm{E}-0.008 \mathrm{P} \\
\text { FRC(L) }-6.7285+0.0631 \mathrm{~A}-0.000519 \mathrm{~A}^{2}+ \\
\quad 0.0603 \mathrm{E}-0.0239 \mathrm{P} \\
\text { RV/TLC } 9.0841+0.4671 \mathrm{~A}+0.0876 \mathrm{E}-0.1518 \mathrm{P}\end{array}$ & & \\
\hline
\end{tabular}

$F=$ femenino, $E=$ estatura, $A=$ años de edad, $P=$ peso, IC $90 \%$ = intervalo de confianza $90 \%$ para límites de normalidad $5-95 \%$,

$\mathrm{RSD}=$ desviación estándar de los residuales en escala logarítmica.

2. Menos de $150 \mathrm{~mL}$ de diferencia entre los dos mayores valores de $\mathrm{VC}{ }^{4}$

k. El registro de sRaw puede realizarse antes de la medición de FRCpleth en las respiraciones a VT donde se estabilizó el nivel de FRC; o bien, posterior a las mediciones solicitando respiración relajada a VT hasta obtener 5 curvas sRaw que sean regulares y reproducibles en análisis visual.

I. Finalizar la prueba y solicitar al paciente que abandone la cabina.

m. Imprimir los resultados.

\section{Informe de pletismografía}

\section{El informe deberá incluir:}

a. Información suficiente para que el responsable de la interpretación pueda evaluar la calidad de la prueba.

b. Datos demográficos de identificación y antropométricos.

c. Origen de los valores de referencia.

d. Los valores absolutos de los volúmenes y capacidades pulmonares se expresan en litros con dos decimales y en condiciones de presión ambiental y temperatura corporal (BTPS).

e. Los valores absolutos y los porcentajes del predicho de las tres maniobras aceptables que incluya FRCpleth, IC, ERV, VC, TLC y RV.

f. El valor promedio de tres maniobras aceptables para FRCpleth, IC, ERV y TLC (la TLC se calcula como la suma de IC más FRCpleth). El RV es igual a la TLC promedio menos el mayor valor de VC. ${ }^{5}$ Una propuesta de reporte se muestra en la Figura 3.

\section{Limitaciones de la prueba}

a. El estándar internacional no emite recomendaciones específicas respecto a valores de referencia. Una relación de ecuaciones disponibles se encuentra en la Tabla 1.

b. Es posible que la FRC se sobreestime en pacientes con obstrucción grave al flujo aéreo. En esos pacientes, cuando la frecuencia respiratoria es mayor a $1 \mathrm{~Hz}$, la presión alveolar no se transmite en forma adecuada a la boca durante la oclusión, lo que en el cálculo sobreestima el volumen. En ese caso se recomienda que la frecuencia respiratoria durante la maniobra sea $<1$ $\mathrm{Hz}{ }^{1}$

c. La maniobra de ITGV es difícil de realizar en algunos pacientes y requiere cooperación del paciente.

d. El exceso de gas abdominal puede llevar a sobreestimaciones del valor de FRCpleth. En el estudio original de DuBois se realizaron mediciones con gas en cámara gástrica y colon sin que eso modificara significativamente la medición; el efecto del gas abdominal se minimiza cuando la oclusión se realiza en FRC. ${ }^{1,3}$

e. El análisis de resistencias tiene amplia variabilidad entre autores en cuanto a los cálculos a partir de los cuales se emite el valor numérico para describir la gráfica flujo-volumen pletismográfico, por lo que cada centro deberá seleccionar el índice a informar para todos los estudios realizados. ${ }^{1}$

\section{Interpretación de la prueba}

Antes de la interpretación deberá evaluar el grado de calidad de la prueba (Tabla 2). La interpretación de la pletismografía se basa en la valoración de la TLC y el RV (el valor promedio) en relación al valor predicho. La valoración del atrapamiento aéreo puede realizarse con el RV por arriba del LSN, o con los índices RV/TLC e IC/TLC. ${ }^{7}$ La 
pletismografía deberá interpretarse siempre en el contexto de la espirometría (Figura 4).

Se considera una pletismografía normal cuando los valores de TLC se encuentran entre el percentil 5 y 95 (aproximadamente 80 a $120 \%$ del predicho). ${ }^{2,5,7}$ Si el valor de TLC se encuentra por debajo del percentil 5 de la ecuación de referencia $(<80 \%)$ se confirma restricción pulmonar. Se considera leve con valores de TLC entre

Tabla 2: Grados de calidad propuestos para pletismografía.

\begin{tabular}{|c|c|c|l|}
\hline $\begin{array}{c}\text { Gra- } \\
\text { do }\end{array}$ & $\begin{array}{c}\text { Maniobras } \\
\text { aceptables }\end{array}$ & $\begin{array}{c}\text { Repetibilidad } \\
\text { Varianza } \\
\text { FRC }_{\text {pleth }} / \Delta \text { VC } \\
<5 \% /<150 \mathrm{~mL}\end{array}$ & \begin{tabular}{l} 
Comentario \\
\hline A
\end{tabular} Técnicamente $_{\text {muy confiable }}$ \\
\hline B & 3 & $5-10 \%<200 \mathrm{~mL}$ & $\begin{array}{l}\text { Técnicamente } \\
\text { confiable }\end{array}$ \\
\hline C & 2 & $5-10 \%<200 \mathrm{~mL}$ & $\begin{array}{l}\text { Técnicamente } \\
\text { aceptable }\end{array}$ \\
\hline D & 2 & $>10 \%>200 \mathrm{~mL}$ & $\begin{array}{l}\text { Técnicamente } \\
\text { con reserva }\end{array}$ \\
\hline E & 1 & & $\begin{array}{l}\text { Técnicamente } \\
\text { no } \\
\text { recomendable }\end{array}$ \\
\hline F & 0 & & $\begin{array}{l}\text { Técnicamente } \\
\text { no } \\
\text { recomendable }\end{array}$ \\
\hline
\end{tabular}

70-79\% del predicho, moderada entre 60-69\%, moderadamente grave entre $50-59 \%$, grave de $35-49 \%$ p y muy grave con valores $<35 \%$ p., 2,7

Cuando la TLC se encuentra por arriba del LSN (> $120 \%$ p) se considera compatible con hiperinflación pulmonar. En enfermedades obstructivas, la determinación del RV y el índice RV/TLC permite confirmar y graduar el atrapamiento aéreo. Valores por arriba del percentil 95 pero inferiores a $140 \%$ p se consideran como atrapamiento leve; entre $140-170 \%$, grado moderado y $>170 \%$, grave. ${ }^{2} \mathrm{El}$ índice IC/TLC, cuando es $\leq 25 \%$, es un predictor significativo de mortalidad en pacientes con EPOC. ${ }^{2,5,7,18}$ El volumen residual es la medición más variable en pletismografía debido a que depende de otras mediciones (FRCpleth, IC y VC) por lo que valores fuera del rango de referencia deberán interpretarse con precaución en ausencia de otras anormalidades. ${ }^{15}$ La definición de patrón mixto (obstrucción y restricción) requiere de la presencia simultánea de relación FEV1/FVC $<$ LIN y TLC $<$ LIN. ${ }^{7}$

En cuanto a la sRaw, la PC se considera el estándar de oro en la medición de resistencias. Su análisis tiene la ventaja de representar al sistema en condiciones fisiológicas de respiración a VT donde refleja propiedades resistivas y de volumen pulmonar. Puede realizarse desde la edad preescolar. El $70 \%$ de los niños que son capaces de hacer una espirometría, pueden completar una PC de buena calidad. Se ha publicado un estándar para su medición en ese grupo de edad con algunas consideraciones especiales. ${ }^{19,20}$ Su aplanamiento significa que se requiere un mayor cambio de volumen para generar un cambio de flujo. ${ }^{2}$ En cambio
Figura 4:

Algoritmo de diagnóstico para pruebas de mecánica, la interpretación de volúmenes pulmonares se realiza con base en la espirometría.

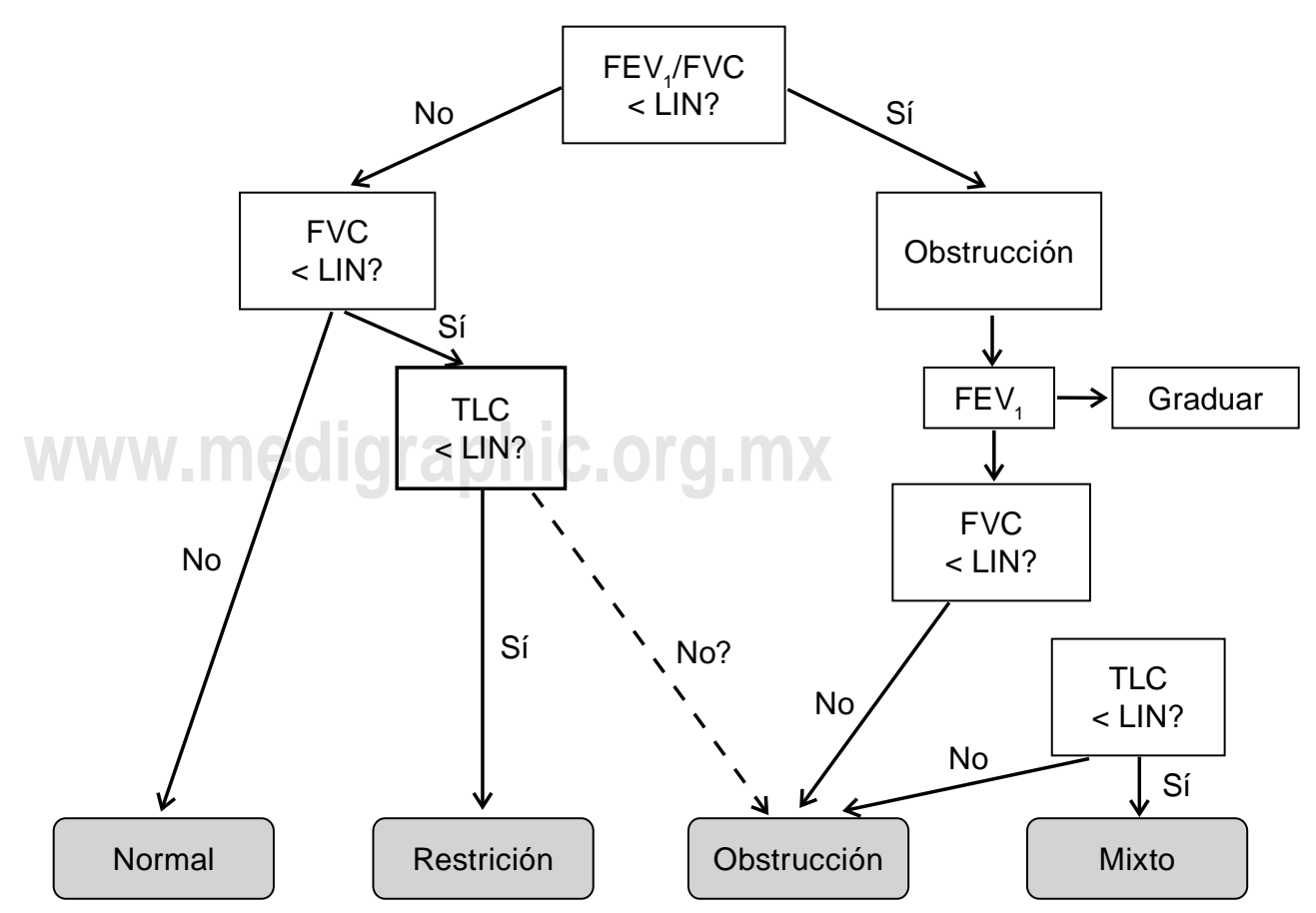




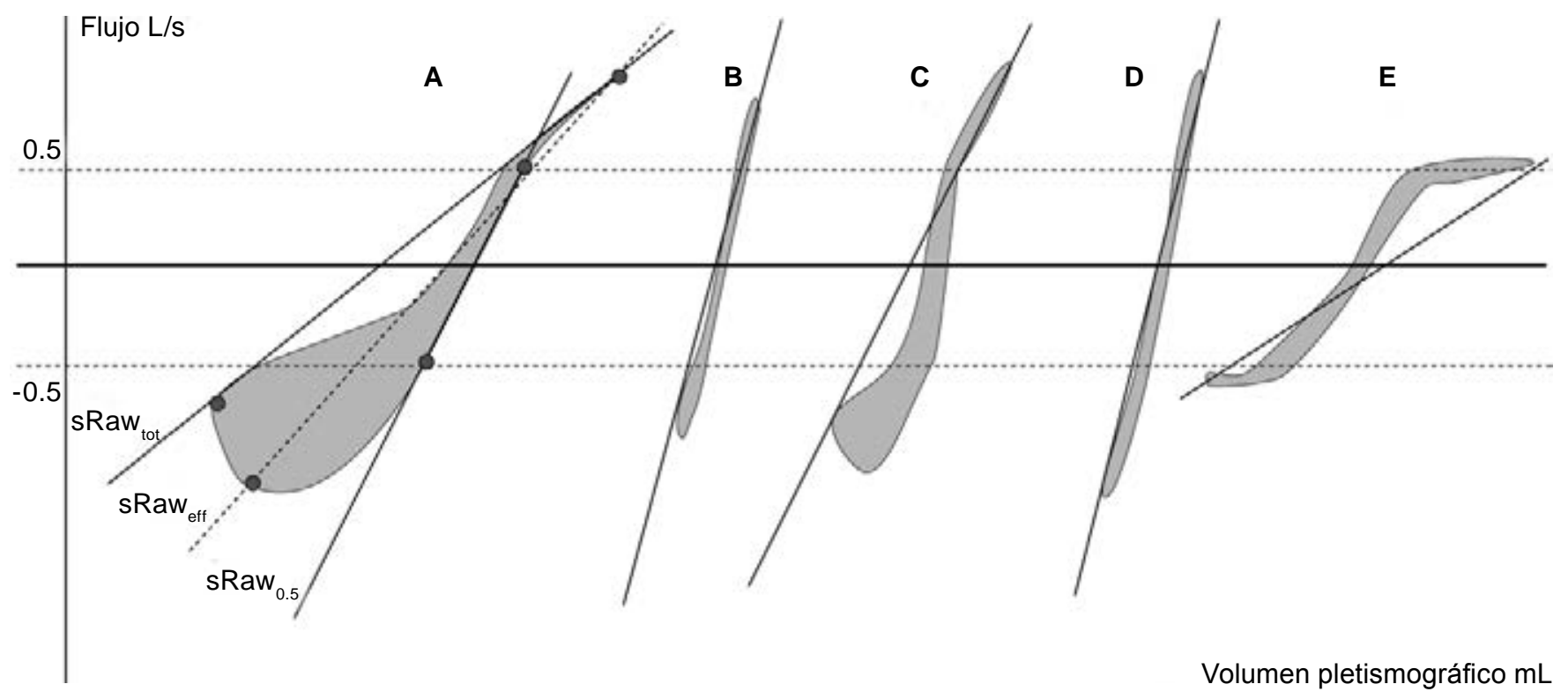

Figura 5: Gráfico flujo-volumen pletismográfico, curva de resistencia en un paciente con obstrucción crónica (A) donde principalmente en la espiración se generan grandes cambios de volumen para pequeños cambios de flujo, se presentan los cálculos propuestos de la pendiente que mejor describe a la curva; sRAWtot pendiente entre los puntos de máximo cambio flujo-volumen para la inspiración y espiración, sRaw ${ }_{0.5}$ pendiente entre los puntos donde la curva atraviesa flujo $+0.5 \mathrm{~L} / \mathrm{s}$ y $-0.5 \mathrm{~L} / \mathrm{s}$, sRaweff pendiente promedio obtenida del análisis dimensional de la curva. Curva de resistencia normal (B) los sujetos sanos requieren pequeños cambios de volumen para generar flujo inspiratorio y espiratorio durante la respiración a volumen corriente los valores de sRaw son muy parecidos en los tres cálculos, curva representativa de pacientes con obstrucción aguda (C), resistencia en paciente con enfermedad restrictiva (D) note cómo se generan altos flujos para pequeños cambios de volumen, sRaw de paciente con estenosis traqueal (E) se requieren amplios cambios de volumen para generar flujos bajos tanto en la inspiración como en la espiración.

la Raw implica ajustar la medición de sRaw por FRCpleth y, por lo tanto, es susceptible a los errores de medición y requerimiento de cooperación asociados con la maniobra de TGV y puede utilizarse a partir de los seis años. ${ }^{15}$ La curva de sRaw es compleja, por lo que múltiples aproximaciones matemáticas han sido propuestas para su análisis (Figura 5). ${ }^{2}$

La sRtot se determina como la pendiente de una línea recta trazada entre los puntos máximos de cambio de volumen inspiratorio y espiratorio. Representa un valor máximo y es sensible a cambios en la vía aérea pequeña; sin embargo, tiene una amplia variabilidad. ${ }^{1}$

La sReff es la resistencia efectiva específica que corresponde a un análisis dimensional de la curva, desde el cual se informa también una pendiente representativa. Su medición es menos variable, se considera que está compuesta principalmente por resistencia de las vías aéreas centrales. ${ }^{1}$

La sRaw ${ }_{0.5}$ consiste en trazar una línea entre los puntos de $+0.5 \mathrm{~L} / \mathrm{s}$ con $-0.5 \mathrm{~L} / \mathrm{s}$, generalmente esta porción de la gráfica es más lineal por lo que tiene menor variabilidad y se considera también representativo de la vía aérea central. Su ajuste por flujo permite eliminar valores artificialmente altos por presencia de turbulencia. ${ }^{1,2}$

Se han propuesto múltiples valores de referencia. La sRawtot en niños es independiente de la talla, género y edad, se aceptan valores de referencia de $1.3 \mathrm{kPa} / \mathrm{s} .{ }^{20}$ Para adultos entre 18 y 65 años se proponen los valores de LSN (> p95) por grupo de edad y género para $\mathrm{sRaw}_{0.5}$, en mujeres < 30 años de $6.18 \mathrm{cmH}_{2} \mathrm{O} / \mathrm{s}$, de los 31-45 años $5.95 \mathrm{cmH}_{2} \mathrm{O} / \mathrm{s}$, entre $46-60$ años $6.35 \mathrm{cmH}_{2} \mathrm{O} / \mathrm{s}$, y para $>60$ años $6.68 \mathrm{cmH}_{2} \mathrm{O} / \mathrm{s}$; $y$ en hombres < 30 años 6.37 $\mathrm{cmH}_{2} \mathrm{O} / \mathrm{s}$, de 31-45 años $6.18 \mathrm{cmH}_{2} \mathrm{O} / \mathrm{s}$, entre 46-60 años $6.78 \mathrm{cmH}_{2} \mathrm{O} / \mathrm{s},>60$ años $6.81 \mathrm{cmH}_{2} \mathrm{O} / \mathrm{s} .{ }^{21}$ Las pruebas de broncodilatación o broncoprovocación consumen tiempo y no están adecuadamente estandarizadas en adultos; en niños se propone una caída $>3$ desviaciones estándar, correspondiente a un cambio de $25 \%$ del predicho. ${ }^{20}$ Para prueba de reto bronquial se considera positivo un aumento en la sReff duplicada y que excede el valor de $2 \mathrm{kPa}$ o bien una caída de sGaw en 35-40\%. ${ }^{2}$

\section{CONCLUSIONES}

La PC es una prueba útil en la caracterización de los patrones funcionales de la mecánica pulmonar. Su medición debe realizarse respetando los estándares internacionales y con un adecuado control de calidad para que los resultados e interpretación sean confiables para el diagnóstico y seguimiento de los pacientes con enfermedad respiratoria. Se requieren valores de referencia provenientes de grandes muestras poblacionales. 


\section{REFERENCIAS}

1. Goldman MD, Smith HJ, Ulmer WT. Whole-body plethysmography. Eur Respir Mon 2005;31:15-43.

2. Criée $\mathrm{CP}$, Sorichter $\mathrm{S}$, Smith HJ, et al. Body plethysmography-its principles and clinical use. Respir Med 2011;105(7):959-971. doi: 10.1016/j.rmed.2011.02.006.

3. DuBois AB, Botelho SY, Bedell GN, Marshall R, Comroe JH Jr. A rapid plethysmographic method for measuring thoracic gas volume: a comparison with a nitrogen washout method for measuring functional residual capacity in normal subjects. J Clin Invest 1956;35(3):322-326.

4. Wanger J, Clausen JL, Coates A, et al. Standardisation of the measurement of lung volumes. Eur Respir J 2005;26(3):511-522.

5. Flesch JD, Dine CJ. Lung volumes: measurement, clinical use, and coding. Chest 2012;142(2):506-510. doi: 10.1378/chest.11-2964.

6. DeCamp MM Jr, Lipson D, Krasna M, Minai OA, McKenna RJ Jr, Thomashow BM. The evaluation and preparation of the patient for lung volume reduction surgery. Proc Am Thorac Soc 2008;5(4):427-431. doi: 10.1513/pats.200707-087ET.

7. Pellegrino R, Viegi G, Brusasco V, et al. Interpretative strategies for lung function tests. Eur Respir J 2005;26(5):948-968.

8. Miller MR, Crapo R, Hankinson J, et al. General considerations for lung function testing. Eur Respir J 2005;26(1):153-161.

9. Miller MR, Hankinson J, Brusasco V, et al. Standardisation of spirometry. Eur Respir J 2005;26(2):319-338.

10. Hepper NG, Black LF, Fowler WS. Relationships of lung volume to height and arm span in normal subjects and in patients with spinal deformity. Dis Chest 1964;37:314-320.

11. Parker JM, Dillard TA, Phillips YY. Arm span-height relationships in patients referred for spirometry. Am J Respir Crit Care Med 1996;154(2 Pt 1):533-536.

12. Chumlea WC, Guo SS, Wholihan K, Cockram D, Kuczmarski RJ, Johnson CL. Stature prediction equations for elderly non-Hispanic white, non-Hispanic black, and Mexican-American persons developed from NHANES III data. J Am Diet Assoc 1998;98(2):137-142.

13. Citado por: Stocks J, Quanjer PH. Reference values for residual volume, functional residual capacity and total lung capacity. ATS Workshop on Lung Volume Measurements. Official Statement of the European Respiratory Society. Eur Respir J 1995;8(3):492-506.

14. Roca J, Burgos F, Barbera JA, et al. Prediction equations for plethysmographic lung volumes. Respir Med 1998;92(3):454-460.

15. Manzke H, Stadlober E, Schellauf HP. Combined body plethysmographic, spirometric and flow volume reference values for male and female children aged 6 to 16 years obtained from «hospital normals». Eur J Pediatr 2001;160(5):300-306.

16. Garcia-Rio F, Dorgham A, Pino JM, Villasante C, Garcia-Quero C, Alvarez-Sala R. Lung volume reference values for women and men 65 to 85 years of age. Am J Respir Crit Care Med 2009;180(11):10831091. doi: $10.1164 / \mathrm{rccm} .200901-01270 \mathrm{C}$.

17. Koch B, Friedrich N, Völzke H, et al. Static lung volumes and airway resistance reference values in healthy adults. Respirology 2013;18(1):170-178. doi: 10.1111/j.1440-1843.2012.02268.x.

18. Casanova C, Cote C, De Torres JP, et al. Inspiratory to total lung capacity ratio predicts mortality in patients with chronic obstructive pulmonary disease. Am J Respir Crit Care Med 2005;171(6):591-597.

19. Vilozni D, Efrati O, Hakim F, Adler A, Livnat G, Bentur L. FRC measurements using body plethysmography in young children. Pediatric Pulmonol 2009;44(9):885-891. doi: 10.1002/ppul.21072.

20. Bisgaard H, Nielsen KG. Plethysmographic measutements of specific airway resistance in young children. Chest 2005;128(1):355-362.

21. Piatti G, Fasano V, Cantarella G, Tarantola C. Body plethysmographic study of specific airway resistance in a sample of healthy adults. Respirology 2012;17(6):976-983. doi: 10.1111/j.14401843.2012.02206.X.

Los autores declaran no tener conflicto de intereses. 